\section{Inibição da lipólise como alvo terapêutico na síndrome metabólica}

\author{
Lipolysis inhibition as therapeutic target in the metabolic syndrome
}

Rennan de Oliveira Caminhotto' ${ }^{1}$ Amanda Baron Campaña ${ }^{1}$, Fabio Bessa Lima ${ }^{1}$

$\mathrm{O}$ grupo de Peter Arner (1), do conceituado Instituto Karolinska em Stockholm, Suécia, foi o primeiro a descrever um fenômeno denominado "resistência à lipólise". Essa resistência se caracteriza pela marcante diminuição da eficácia das catecolaminas em estimular a lipólise em adipócitos de pacientes com síndrome metabólica.

A "resistência à lipólise" ocorre em consequência da menor capacidade de ativação da lipase sensível por hormônios (LHS) via AMPc, assim como pela combinação de múltiplos defeitos em vias adrenérgicas da mobilização dos lipídios, como a redução da expressão de receptores $\beta$-adrenérgicos. Tais dados foram confirmados e aprofundados em outras importantes publicações, nas quais também foi descrita menor expressão de lipases (2-4). Além disso, esses estudos estabeleceram correlações entre sensibilidade à insulina sistêmica e o número de receptores $\beta_{2}$-adrenérgicos, bem como entre taxas máximas de lipólise e tolerância à glicose, demonstrando que quão menor a capacidade em realizar lipólise maior é o quadro de resistência à insulina e intolerância à glicose (1).

Assim sendo, é razoável a hipótese de que, na síndrome metabólica, a dificuldade de mobilizar lipídios em adipócitos poderia, então, contribuir para o aumento da massa adiposa, agravando o quadro de obesidade e, consequentemente, reduzindo a sensibilidade à insulina.

Entretanto, em trabalho recente (5) no qual participam Peter Arner e Dominique Langin, do Instituto de Doenças Metabólicas e Cardiovasculares da Universidade de Toulouse, França, o conhecimento da participação dos mecanismos de regulação da lipólise na obesidade e síndrome metabólica adquiriu uma nova temática.

Segundo os autores, sabe-se que, em excesso, os ácidos graxos podem induzir resistência à ação da insulina. Neste estudo, a observação clínica da variação natural da lipólise (isto é, da lipólise não estimulada) em células de gordura de humanos revelou que a elevada taxa de lipólise basal está associada com baixa sensibilidade à insulina. Demonstrou-se, ainda, que a menor ação da enzima LHS, seja por modificações genéticas ou por inibição farmacológica, resulta em melhora da sensibilidade à insulina em ratos. Uma série de estudos em camundongos e em células de gordura humana demonstrou que, ao atenuar a capacidade lipolítica, a síntese de novos ácidos graxos a partir da glicose era ativada. Essa via metabólica foi recentemente proposta (6) como um dos principais determinantes da sensibilidade sistêmica à insulina.

Tais dados levaram a crer que a inibição parcial da lipólise é uma estratégia plausível no tratamento da resistência à insulina associada à síndrome metabólica, o que parece paradoxal quando comparado aos trabalhos prévios do mesmo grupo. Nossa experiência em metabolismo de adipócitos nos permite explicar esse aparente paradoxo.
Laboratório de Fisiologia do Tecido Adiposo, Departamento de Fisiologia e Biofísica, Instituto de Ciências Biomédicas, Universidade de São Paulo (USP), São Paulo, SP, Brasil

Correspondência para: Fabio Bessa Lima

Universidade de São Paulo, Instituto de Ciências Biomédicas Av. Prof. Lineu Prestes, 1524, Ed. Biomédicas I, sala 131 05508-900 - São Paulo, SP, Brasil fabio@icb.usp.br

Recebido em 22/Mar/2013 Aceito em 29/Maio/2013 
Durante a síndrome metabólica, atividade lipolítica basal de adipócitos geralmente está aumentada (7). Ainda, como já bem descrito, o tecido adiposo visceral, presente em quantidade abundante na síndrome metabólica, possui uma atividade metabólica mais intensa que outros coxins. Assim, a maior atividade lipolítica desse coxim contribui com importante aumento de ácidos graxos livres na circulação. De tal modo, uma redução da atividade lipolítica reduziria o aporte de ácidos graxos livres à circulação, o que corrobora o benefício metabólico encontrado nos resultados do estudo supracitados (5). Portanto, em quadros de adiposidade visceral, o bloqueio parcial da lipólise parece ser uma alternativa benéfica.

Por outro lado, trabalhos prévios do grupo (3) identificaram em obesos (IMC $37 \pm 5 \mathrm{~kg} / \mathrm{m}^{2}$, grupo classicamente intolerante à glicose) defeitos na lipólise estimulada e expressão de lipases de células provenientes do tecido adiposo subcutâneo, o maior depósito corporal de gordura. Dessa forma, nesse quadro de resistência à insulina associado à obesidade, a inibição parcial da lipólise pode ser uma alternativa discutível, uma vez que a perda de peso desejável poderia ser prejudicada.

Em outra publicação (8), Langin discorre sobre propostas de abordagens farmacológicas para a regulação da lipólise no tratamento da síndrome metabólica. Em uma das propostas, a abordagem farmacológica era a inibição parcial da lipólise e a melhora da sensibilidade à insulina em modelos experimentais.

A outra proposta consiste em estimular a lipólise e oxidação dos ácidos graxos, o que permitiria uma abordagem completa do quadro de adiposidade e resistência à insulina. Dentro dessa perspectiva, vem ganhando destaque o emprego de peptídeos atriais natriuréticos que, além de estimular lipólise (9), estão associados à programação termogênica em adipócitos brancos e marrons, por intenso estímulo da biogênese mitocondrial, estimulando, assim, a oxidação de gordura e diminuição do peso corporal de animais (10).

Por fim, o controle fino da atividade lipolítica nos diferentes coxins adiposos destaca-se como uma importante ferramenta para o controle da sensibilidade à insulina em morbidades como obesidade e síndrome metabólica, consolidando, mais uma vez, a hipótese de que o controle da atividade metabólica de adipócitos constitui-se em um importante objeto de intervenção terapêutica no tratamento de doenças metabólicas.

Agradecimentos: à Fundação de Amparo à Pesquisa do Estado de São Paulo (Fapesp), ao Conselho Nacional de Desenvolvimento Científico e Tecnológico $(\mathrm{CNPq})$ e à Coordenação de Aperfeiçoamento de Pessoal de Nível Superior (Capes), pelos auxílios e pelas bolsas concedidas no momento.

Declaração: os autores declaram não haver conflitos de interesse científico neste estudo.

\section{REFERÊNCIAS}

1. Reynisdottir S, Ellerfeldt K, Wahrenberg $H$, Lithell H, Arner P. Multiple lipolysis defects in the insulin resistance (metabolic) syndrome. J Clin Invest. 1994;93(6):2590-9.

2. Large $V$, Reynisdottir $S$, Langin $D$, Fredby $K$, Klannemark M, Holm C, et al. Decreased expression and function of adipocyte hormone-sensitive lipase in subcutaneous fat cells of obese subjects. J Lipid Res. 1999;40(11):2059-66.

3. Langin D, Dicker A, Tavernier G, Hoffstedt J, Mairal A, Ryden M, et al. Adipocyte lipases and defect of lipolysis in human obesity. Diabetes. 2005;54(11):3190-7.

4. Jocken JW, Langin D, Smit E, Saris WH, Valle C, Hul GB, et al. Adipose triglyceride lipase and hormone-sensitive lipase protein expression is decreased in the obese insulin-resistant state. J Clin Endocrinol Metab. 2007;92(6):2292-9.

5. Girousse A, Tavernier G, Valle C, Moro C, Mejhert N, Dinel AL, et al. Partial inhibition of adipose tissue lipolysis improves glucose metabolism and insulin sensitivity without alteration of fat mass. PLoS Biol. 2013;11(2):e1001485.

6. Herman MA, Peroni OD, Villoria J, Schon MR, Abumrad NA, Bluher $M$, et al. A novel ChREBP isoform in adipose tissue regulates systemic glucose metabolism. Nature. 2012;484(7394):333-8.

7. Gaidhu MP, Anthony NM, Patel P, Hawke TJ, Ceddia RB. Dysregulation of lipolysis and lipid metabolism in visceral and subcutaneous adipocytes by high-fat diet: role of ATGL, HSL, and AMPK. Am J Physiol Cell Physiol. 2010;298(4):C961-71.

8. Langin D. Adipose tissue lipolysis as a metabolic pathway to define pharmacological strategies against obesity and the metabolic syndrome. Pharmacol Res. 2006;53(6):482-91.

9. Sengenes C, Berlan M, De Glisezinski I, Lafontan M, Galitzky J. Natriuretic peptides: a new lipolytic pathway in human adipocytes. FASEB J. 2000;14(10):1345-51.

10. Bordicchia M, Liu D, Amri EZ, Ailhaud G, Dessi-Fulgheri P, Zhang $C$, et al. Cardiac natriuretic peptides act via p38 MAPK to induce the brown fat thermogenic program in mouse and human adipocytes. J Clin Invest. 2012;122(3):1022-36. 